\title{
EVolution of A DUSt Shell Along A Stellar Post-AgB
}

TRACK

\author{
H. MARTEN ${ }^{1}$, R. SZCZERBA ${ }^{2}$ AND T. BLÖCKER ${ }^{1}$ \\ ${ }^{1}$ Institut für Theoretische Physik und Sternwarte der Universität Kiel \\ Olshausenstr. 40, D-2300 Kiel, Germany \\ ${ }^{2}$ Copernicus Astronomical Center, Laboratory of Astrophysics \\ ul. Chopina 12/18, 87-100 Toruń, Poland
}

We study the spectral appearance of an evolving post-AGB object by means of twofluid radiation hydrodynamics (gas and dust) in spherical symmetry. The stellar parameters as well as mass-loss rates are assumed to vary consistently with the stellar model calculations of Blöcker (1989, Diplom Thesis, University of Kiel) for a $3 \mathrm{M}_{\odot}$ sequence, resulting in a $0.605 \mathrm{M}_{\odot}$ remnant. The expelled mass is assumed to consist of spherical astronomical silicates with grain radii of $0.1 \mu \mathrm{m}$ and an initial dust-to-gas mass ratio of $7.5 \cdot 10^{-3}$. For further details on the computational method we refer to Szczerba \& Marten (1992, in: Proc. of the ESO/CTIO Workshop, Mass Loss on the AGB and Beyond, La Serena 1992, in press).

Following the last $30000 \mathrm{yrs}$ of AGB evolution along Blöckers $3 \mathrm{M}_{\odot}$ sequence with increasing mass-loss rates and an initial wind velocity of $5 \mathrm{~km} / \mathrm{sec}$, the star finally becomes totally obscured by a thick dust shell which expands with a final velocity of about $15 \mathrm{~km} / \mathrm{sec}$. In the calculations of Blöcker, the heavy mass loss of the red giant is eventually smoothly decreased between the stellar radial fundamental pulsation periods of 100 and $50 \mathrm{~d}$ (see also Blöcker \& Schönberner, 1990, A\&A 240, L11). During this short phase of only about $200 \mathrm{yrs}$ the stellar continuum reappeares as a bump shortwards of the $\mathrm{L}$ photometric band. The decreasing mass loss (density) reduces the frictional coupling between dust and gas, and the dust is accelerated to about $150 \mathrm{~km} / \mathrm{sec}\left(\mathrm{v}_{\text {gas }} \approx 50 \mathrm{~km} / \mathrm{sec}\right)$. As a result, the contribution of newly formed (hot) dust from this high velocity/low density wind region to the overall spectra during the post-AGB phase is much smaller than expected from the quasi-stationary approach of other authors. Consequently, our model tracks with and without further dust condensation show a very similar behaviour in the $\log \left(\lambda F_{\lambda}(60) / \lambda F_{\lambda}(25)\right)$ versus $\log \left(\lambda F_{\lambda}(25) / \lambda F_{\lambda}(12)\right)$ plane with a maximum difference in their colors of only about $0.4 \mathrm{dex}$. At a post-AGB age of about $600 \mathrm{yrs}\left(\mathrm{T}_{\mathrm{eff}} \approx\right.$ $7500 \mathrm{~K}$ ), our model sequences in the IRAS diagram start to deviate from what is expected for a simply expanding and cooling shell. Instead of evolving downwards, describing a big loop until the colors of black bodies with the corresponding stellar temperature(s) are reached, the dust shells are re-heated due to the growing stellar temperatures and the models start to proceed to the upper right.

Both, the non-linear dynamical effects of the dust acceleration as well as consistent evolutionary changes of the stellar parameters and mass-loss rates must be taken into consideration in order to understand the exact course of the mass loss and its influence on the timescales for post-AGB evolution. 